\title{
Quantum thermodynamics with local control
}

\author{
J. Lekscha,,${ }^{1,2,3}$ H. Wilming, ${ }^{1}$ J. Eisert, ${ }^{1}$ and R. Gallego ${ }^{1}$ \\ ${ }^{1}$ Dahlem Center for Complex Quantum Systems, Freie Universität Berlin, 14195 Berlin, Germany \\ ${ }^{2}$ Potsdam Institute for Climate Impact Research, 14473 Potsdam, Germany \\ ${ }^{3}$ Department of Physics, Humboldt-Universität zu Berlin, 12489 Berlin, Germany
}

(Received 2 February 2017; revised manuscript received 18 October 2017; published 27 February 2018)

\begin{abstract}
We investigate the limitations that emerge in thermodynamic tasks as a result of having local control only over the components of a thermal machine. These limitations are particularly relevant for devices composed of interacting many-body systems. Specifically, we study protocols of work extraction that employ a many-body system as a working medium whose evolution can be driven by tuning the on-site Hamiltonian terms. This provides a restricted set of thermodynamic operations, giving rise to alternative bounds for the performance of engines. Our findings show that those limitations in control render it, in general, impossible to reach Carnot efficiency; in its extreme ramification it can even forbid to reach a finite efficiency or finite work per particle. We focus on the one-dimensional Ising model in the thermodynamic limit as a case study. We show that in the limit of strong interactions the ferromagnetic case becomes useless for work extraction, while the antiferromagnetic case improves its performance with the strength of the couplings, reaching Carnot in the limit of arbitrary strong interactions. Our results provide a promising connection between the study of quantum control and thermodynamics and introduce a more realistic set of physical operations well suited to capture current experimental scenarios.
\end{abstract}

DOI: 10.1103/PhysRevE.97.022142

\section{INTRODUCTION}

Recently, notions of quantum thermodynamics, and in particular questions on how much work can be extracted in systems in which quantum effects are expected to be relevant, have received a lot of attention. Much focus has been put on understanding fundamental limits on the amount of work that can be extracted from a single quantum system prepared in a state out of thermal equilibrium. This research program is two-pronged: On the one hand, there is an emphasis on identifying the laws of quantum thermodynamics [1-6], as primitives from which macroscopic thermodynamics can be derived. On the other hand, a significant body of literature is concerned with characterizing the behavior of realistic physical devices operating at a scale where quantum effects become relevant $[4,7-10]$ as well as incorporating control limitations over general sets of thermodynamics operations [11-13].

One of the key aspects in these efforts is to understand how quantum thermodynamic notions precisely behave under composition of subsystems. This comprises the study of the role of correlations between subsystems [13-17], of the scalability of quantum engines [18-20], and of the emergence of thermodynamics from a more fundamental quantum description of its constituents $[9,21]$. This body of literature focuses on composite systems that, although often displaying classical or even quantum correlations between its subsystems reflecting a past interaction, do not interact, or at least not beyond the weak-coupling regime.

In this work, we contribute to filling this gap by focusing on the study of work extraction with many-body systems with possibly strong couplings between subsystems. An important question that emerges when dealing with such strongly interacting systems is that of determining the possible transformations that one can induce in the state of the compound by having local control only [22]. A reasonable setting for a many-body system is one where the experimenter will be able to apply and vary external fields that will control the on-site Hamiltonian terms; at the same time, the interaction terms between the subsystems cannot be modified at will. This constitutes a limitation on the set of reachable Hamiltonians and consequently on the possible dynamics that the system may undergo. This is a most natural setting: The field of quantum control (QC) can be seen as largely studying the type of dynamics precisely in such a setting [23-28]. Here, we explore the surprising ramifications of local control for the performance of thermodynamic tasks. We believe that the identification of this physically reasonable class of thermodynamic state transformations constitutes an important aspect of this work in its own right.

We introduce interactions and limitations on control into the problem of work extraction by considering the situation of an engine operating with a many-body system as a working medium and two thermal baths at different temperatures. The working medium has some fixed interactions of arbitrary strength among its constituents. The engine is operated by applying some time-dependent external fields and putting the working medium in contact with either of the baths. For this general scenario, we investigate the limitations emerging due to the lack of global control, simply by comparing with the usual bounds provided by the second law. Those limitations will affect the efficiency of the engine as a function of the interactions and the size of the many-body system.

As a first result, we find a fully general expression describing the corrections to the Carnot efficiency as a function of the interactions, showing that it is impossible, in general, to achieve Carnot efficiency exactly and interactions lead to 
irreversibility in the thermodynamic sense. Also, by employing results from the theory of $\mathrm{QC}$, we show that our bounds are saturated for generic interactions. We then elaborate on bounds for the 1D Ising model as a case study. Surprisingly, this model displays a strikingly different behavior for the antiferromagnetic and the ferromagnetic regimes. The former case allows for a finite work output per particle, as well as reaching Carnot efficiency in the limit of very strong couplings. The latter displays an opposite behavior, where very strong couplings imply vanishing work per particle and efficiency. This shows that limitations due to local control crucially affect the scalability or performance in the macroscopic limit. Indeed, ranging from the two extreme behaviors of allowing for Carnot efficiency or preventing one to extract any work whatsoever.

\section{SET-UP AND OPERATIONS}

We consider a thermal machine composed by a working medium and two baths at different temperatures. The working medium is taken to be a many-body system composed of $N$ subsystems. The machine is operated by performing two kinds of operations.

First, one can change the Hamiltonian of the working medium over time. The working medium is hence described at time $t$ by the pair $[\rho(t), H(t)]$ of a quantum state and a time-dependent Hamiltonian,

$$
H(t)=H_{\mathrm{ext}}(t)+H_{\mathrm{int}} .
$$

The term $H_{\text {ext }}(t)$ represents the external fields $H_{\text {ext }}(t)=$ $\sum_{j}^{N} H^{(j)}(t)$ that can be varied with time, where $H^{(j)}=\mathbb{1}_{1} \otimes$ $\cdots \otimes h^{(j)}(t) \otimes \cdots \otimes \mathbb{1}_{N}$ is a Hamiltonian acting on the $j$ th subsystem only. Clearly, it is of physical relevance to consider the special case in which $h^{(j)}(t)=h(t) \forall j$, that is, where the external fields act equally in all subsystems. This will be indeed the case considered in our case studies, but we keep here the discussion as general as possible. $H_{\mathrm{int}}$, in contrast, is an arbitrary time-independent interaction between the subsystems. The form of the interactions between the constituents of the working medium will be crucial in this step, because they shape the limitations on the set of Hamiltonians that can be chosen. This limitation is the most natural when dealing with many-body systems that can be affected by controlled external fields, although their interactions are not accessible to the experimenter. The time evolution between times $t_{1}$ and $t_{2}$ of the working medium under Hamiltonian Eq. (1) results in a transition,

$$
\left[\rho\left(t_{1}\right), H\left(t_{1}\right)\right] \mapsto\left[U\left(t_{1}, t_{2}\right) \rho\left(t_{1}\right) U^{\dagger}\left(t_{1}, t_{2}\right), H\left(t_{2}\right)\right],
$$

where $U\left(t_{1}, t_{2}\right)$ is the unitary evolution induced by the timedependent Hamiltonian Eq. (1). This transition results in a change of the total mean energy of the working medium, which is the expected work $W$ extracted in the process, so that

$$
W\left(t_{1}, t_{2}\right)=\operatorname{tr}\left[\rho\left(t_{1}\right) H\left(t_{1}\right)\right]-\operatorname{tr}\left[\rho\left(t_{2}\right) H\left(t_{2}\right)\right],
$$

where $\rho\left(t_{2}\right)=U\left(t_{1}, t_{2}\right) \rho\left(t_{1}\right) U^{\dagger}\left(t_{1}, t_{2}\right)$. Given that the current value of the time employed will not be relevant for work and efficiency considerations, we can just describe the processes by $W^{i, i+1}:=W\left(t_{i}, t_{i+1}\right)$ and equivalenty $\left(\rho^{i}, H^{i}\right):=$ $\left[\rho\left(t_{i}\right), H\left(t_{i}\right)\right]$.
Second, we will consider another kind of operations that represent the thermal contact between the working system and a thermal bath at inverse temperature $\beta$. We will assume that throughout the protocol there are two different baths available, one hot bath and one cold bath, with inverse temperatures given by $\beta_{h}$ and $\beta_{c}$, respectively. These operations have the effect of bringing the working medium to the Gibbs state of the corresponding Hamiltonian. That is,

$$
\left(\rho^{i}, H^{i}\right) \mapsto\left[\omega\left(H^{i}, \beta\right), H^{i}\right],
$$

where $\omega\left(H^{i}, \beta\right)=\exp \left(-\beta H^{i}\right) / Z_{\beta}\left(H^{i}\right)$ and the partition function is given by $Z_{\beta}\left(H^{i}\right)=\operatorname{tr}\left[\exp \left(-\beta H^{i}\right)\right]$. To simplify the notation we will simply denote Gibbs states by $\omega_{h}^{i}:=\omega\left(H^{i}, \beta_{h}\right)$ and equivalently for the cold bath. The transformation given by Eq. (4) occurs when placing a sufficiently weak interaction between the working medium and the thermal bath and does not require any work investment. These two kinds of steps are repeated at will to perform a protocol. Importantly, a sequence of arbitrarily small changes of the Hamiltonian of the form of Eq. (2), followed each by a thermal contact Eq. (4) produces an isothermal path, that is, a trajectory of Hamiltonians where the system is at all times in the corresponding Gibbs state.

\section{Engine cycles}

The two kinds of operations Eqs. (1) and (4) described above are combined arbitrarily in a protocol yielding a total expected work given by $W=\sum_{i}^{n} W^{i, i+1}$, where $i$ sums over all the steps in which a time evolution under the time-dependent Hamiltonian has occurred. The protocol cyclic, so that after the $n$ steps that change the Hamiltonian we return to the initial Hamiltonian, that is, $H^{n}=H^{0}$. During the contacts with the thermal baths resulting in Eq. (4) the bath and working medium exchange heat. The heat provided by the hot thermal bath is given by $Q_{h}=\sum_{i}^{k}\left[\operatorname{tr}\left(\omega_{h}^{i} H^{i}\right)-\operatorname{tr}\left(\rho^{i} H^{i}\right)\right]$, where $i$ sums over the steps of the protocol where a thermal contact with the hot thermal bath is implemented. Finally, the efficiency of the engine performing a given cycle is defined as $\eta=W /\left|Q_{h}\right|$. We will now study limitations on the maximal efficiency achievable given as a function of the interaction term $H_{\text {int }}$.

\section{LIMITATIONS TO CARNOT EFFICIENCY}

As it is clear from basic considerations in (quantum) thermodynamics, the optimal efficiency is reached by reversible protocols. This can be easily appreciated in the Carnot cycle as depicted in Fig 1. Within the framework of phenomenological thermodynamics the working medium (say, a gas in a piston) is described by its entropy and temperature. It is necessary, to perform a Carnot cycle, that an adiabatic compression and expansion of the gas in the piston can alter its temperature at will within the range given by the two baths. That is, if one has a gas at temperature $T_{c}$ (after contact with the cold bath) one can compress rapidly the piston to increase its temperature to $T_{h}$, the latter being the temperature of the hot bath. The temperature will increase monotonically with the strength of the compression. Hence, to reach $T_{h}$ one only needs to compress the gas sufficiently.

The idealization of a Carnot engine is similar when we deal with a microscopic working medium. In this case, it will 
(a) $S$

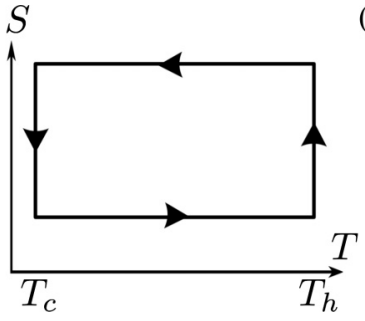

(b) $S$

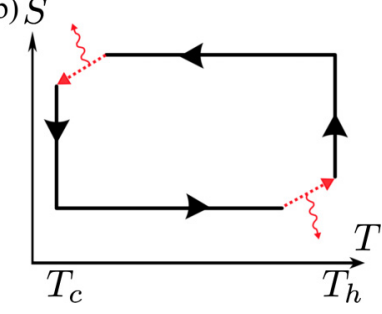

FIG. 1. (a) A Carnot cycle in an entropy-temperature diagram. When the working-medium is put in contact with the heat bath, it is already at the same temperature as the bath. Hence, no heat flows from the heat bath until one starts the isothermal expansion. (b) If the bath's temperature cannot be reached by an adiabatic expansion and compression, then there is an unavoidable dissipation (red arrows) reducing the efficiency.

not be described by the coarse-grained variables entropy and temperature, but with the pair $\left(\rho^{i}, H^{i}\right)$ of the quantum state $\rho^{i}$ and the Hamiltonian $H^{i}$, taking different configurations over the protocol.

Similar to the usual Carnot cycle of Fig. 1, maximal efficiency is achieved when the protocol is reversible. This requires that the working medium, after contact with the cold heat bath in state $\omega_{c}^{i}(H)$ can be transformed by an adiabatic process-in this case one of the form Eq. (2) - into a state $\omega_{h}^{i}\left(H^{\prime}\right)$. In other words, one must be able to "compress" the cold working medium until it reaches the temperature of the hot bath. This is always possible for a gas in a piston, but as we will show it is not possible for many-body systems evolving under Hamiltonians of the form of Eq. (1). This insight constitutes the main result of this manuscript and it is responsible for the impossibility of reaching Carnot efficiency, which is captured precisely in the following set of results.

\section{A. General bound}

We given now an upper bound to the efficiency that can be obtained by any protocol combining operations Eqs. (2) and (4).

Theorem 1 (General bound). All protocols that combine operations Eqs. (2) and (4) have an efficiency bounded as

$$
\eta \leqslant 1-\frac{T_{c}}{T_{h}}\left[\frac{\Delta S^{B, D}+\min _{U} D\left(U \omega_{h}^{B} U^{\dagger} \| \omega_{c}^{C}\right)}{\Delta S^{B, D}-\min _{V} D\left(V \omega_{c}^{D} V^{\dagger} \| \omega_{h}^{A}\right)}\right],
$$

where $\omega_{i}^{J}:=\omega\left(H^{J}, \beta_{i}\right)$ and $H^{J}$ are arbitrary Hamiltonians of the form of Eq. (1); $\Delta S^{B, D}:=S\left(\omega_{h}^{B}\right)-S\left(\omega_{c}^{D}\right)$, where $S$ is the von Neumann entropy, $D(\cdot \| \cdot)$ is the relative entropy; $U$ and $V$ are unitary transformations that can be induced by any trajectory of $H(t)$ as in Eq. (1).

The main idea behind the proof, which is given in Sec. V A, is the insight discussed above that it is, in general, impossible to bring by an adiabatic process $\omega_{c}(H)$ into a state of the form $\omega_{h}\left(H^{\prime}\right)$ when the set of Hamiltonians is restricted to Eq. (1). This implies that a Carnot protocol cannot be performed which in turn produces the corrections in Eq. (5). The proof strategy is to first show that a family of protocols combining adiabatic and isothermal transformations are optimal and then minimize the irreversible dissipation for this family of protocols (see

Sec. V A 1). Furthermore, our proof is constructive in the sense that it provides the optimal protocol achieving the efficiency of Eq. (5). We note, however, that the bound Eq. (5) is general in the sense that it upper bounds the efficiency for any conceivable protocol combining arbitrarily adiabatic transformations and contacts with the baths in any order and for any number of steps. Also, we point out that a similar bound can be obtained for restrictions in control other than Eq. (1). We focus on this case here for simplicity of presentation.

It is also worth discussing simplified bounds on the efficiency that can be also saturated in two relevant regimes. First, note that if $\left[H_{\mathrm{ext}}(t), H_{\mathrm{int}}\right]=0 \forall t$, then any unitary $U$ generated by a trajectory of $H(t)$ will be such $\left[U, \omega_{i}^{J}\right]=0$. In this case, Eq. (5) is replaced by

$$
\eta \leqslant 1-\frac{T_{c}}{T_{h}}\left[\frac{\Delta S^{B, D}+D\left(\omega_{h}^{B} \| \omega_{c}^{C}\right)}{\Delta S^{B, D}-D\left(\omega_{c}^{D} \| \omega_{h}^{A}\right)}\right] .
$$

This latter bound applies also if one considers arbitrary Hamiltonians $H(t)$ but limits instead the set of operations Eq. (2) to Hamiltonian quenches, where $U\left(t_{1}, t_{2}\right)=\mathbb{1}$.

Second, consider the case where a trajectory of $H(t)$ can induce any possible global unitary transformations $U$ and $V$. In this case the bound Eq. (5) is replaced by

$$
\eta \leqslant 1-\frac{T_{c}}{T_{h}}\left[\frac{\Delta S^{B, D}+D^{\downarrow}\left(\omega_{h}^{B} \| \omega_{c}^{C}\right)}{\Delta S^{B, D}-D^{\downarrow}\left(\omega_{c}^{D} \| \omega_{h}^{A}\right)}\right],
$$

where $D^{\downarrow}(\cdot \| \cdot)$ is the relative entropy defined as $D^{\downarrow}(\rho \| \sigma):=$ $\sum_{m} \rho_{m} \ln \left(\rho_{m} / \sigma_{m}\right)$, with $\left\{\rho_{m}\right\}$ and $\left\{\sigma_{m}\right\}$ being the set of eigenvalues of $\rho$ and $\sigma$, respectively, both ordered in nonincreasing order. The bound Eq. (7) follows from majorization arguments (see Sec. VB) and its a universal bound on the efficiency [it is larger than the right-hand side of Eq. (5)]. Indeed, Theorem 1 allows us to recover the usual Carnot efficiency in the case of vanishing interactions, since in this case the correction terms can be made zero by appropriate choice of local fields (see Sec. VC). Finally, we note that the correction terms in the optimal efficiency scale extensively for local many-body systems. Hence, a similar bound holds when we consider for the efficiency the work density and heat density instead of the total work and total heat.

\section{B. Saturating the bound for generic interactions}

Previously we have seen that the maximal possible value of the efficiency as a function of the interactions $H_{\text {int }}$ is given by Eq. (7). It is a natural task to establish conditions where it can be saturated. Here we argue that the bound Eq. (7) can be saturated generically. For this, we rely on results in the field of quantum control showing that under a Hamiltonian of the form of Eq. (1), any unitary in the Lie-algebra generated by $H_{\text {int }}$ and the locally controllable fields $H^{(i)}(t)$ can be approximated arbitrarily well [23-28]. For generic, locally interacting Hamiltonians, this Lie-algebra is the full special unitary Lie-algebra on the Hilbert-space and thus any global unitary can in principle be approximated arbitrarily well. Indeed, often one does not even need to control the on-site field of all the spins. For example, in a spin-chain with Heisenberg-like interactions, control over a 


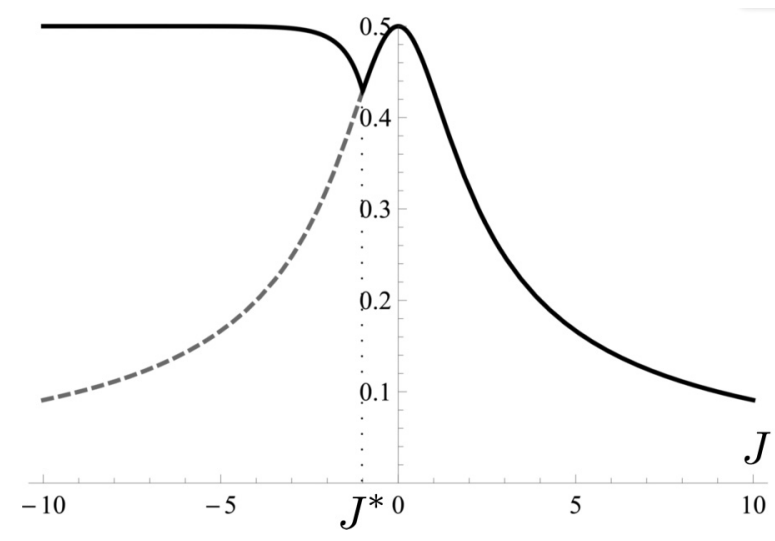

FIG. 2. Efficiency at maximum work density (black) for the Ising model as a function of $J$ in the thermodynamic limit for the parameters $\beta_{h}=0.5, \beta_{c}=1$. The gray dashed line shows a protocol that is independent of $J$. Carnot efficiency is 0.5 .

single spin is in principle sufficient to implement any unitary evolution [26,28].

\section{CASE STUDY: ISING MODEL}

We focus now on the Ising model as a case study, it being instructive and sharing all the main features discussed here. The goal is to study the limitations to Carnot derived in Theorem 1 from a quantitative perspective. We will show that the corrections to Carnot influence dramatically the feasibility of work extraction protocols.

We consider work extraction from a many-body spin system with nearest neighbor Ising Hamiltonian, for which Eq. (1) takes the form

$$
H_{\mathrm{I}, N}(t)=-h(t) \sum_{j=1}^{N} \sigma_{z}^{(j)}-J \sum_{j=1}^{N} \sigma_{z}^{(j)} \sigma_{z}^{(j+1)} .
$$

Here, $\sigma_{z}^{(j)}$ denotes the Pauli $Z$ matrix acting at spin $j, h(t)$ is a tunable magnetic field, and $J$ a fixed interaction strength. Note that we are assuming that the external field $h(t)$ is translational invariant and commutes with the interaction. Therefore, the unitaries $U, V$ in Eq. (6) are in fact identities. We thus obtain a set of operations less general as the one given by Eq. (1), but at the same time it fairly represents a more realistic situation than applying different external fields to each microscopic subsystems. We assume periodic boundary conditions, i.e., $\sigma_{z}^{(N+1)}=\sigma_{z}^{(1)}$. As the Hamiltonian Eq. (8) is diagonal, it is equivalent to the classical Ising model Hamiltonian with $\sigma_{z}^{(j)}=$ $\sigma^{(j)} \in\{-1,1\}$ denoting spin up or down, respectively. Thus, we will be able use the well known results about the partition function of the Ising model when studying work extraction. Using the partition function and the bounds of Theorem 1 one can compute the efficiency at maximum work density as function of $J$. This is shown in Fig. 2 in the thermodynamic limit.

There are three relevant aspects of the efficiency plotted in Fig. 2 that we can derive analytically and that clearly exemplify the behavior of the bound Eq. (5): (i) in the limit of strong antiferromagnetic interactions $J \rightarrow-\infty$ one can reach Carnot efficiency, (ii) in the limit of strong ferromagnetic interactions (a) $\rho$

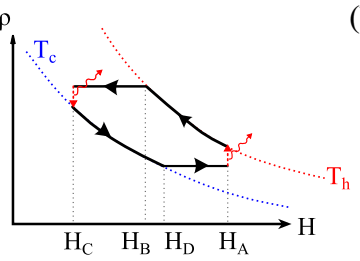

(b) $\rho$

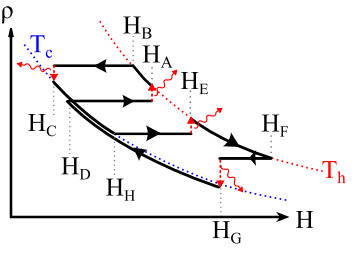

FIG. 3. (a) Carnot-like protocol; (b) protocol that is not Carnotlike. The figure shows that a non-Carnot-like protocol can always be understood as a concatenation of small Carnot-like protocols connected by isothermals at the cold bath.

$J \rightarrow \infty$ efficiency drops to zero, and (iii) at the value $J=J^{*}$, the efficiency changes its behavior abruptly. All these three points can be explained analytically relying on considerations about the ground-state degeneracy of the (anti)ferromagnetic regimes and the resulting optimal protocols (see Sec. VI).

Regarding point (i) we construct in Sec. VIB a simple protocol that reaches Carnot in the limit of $J \rightarrow-\infty$. Furthermore, this protocol is able to reach a finite work output per particle and cycle. This protocol works also in the thermodynamic limit, hence showing that one can build effective engines with macroscopic strongly correlated antiferromagnetic spin chains.

The limit of $J \rightarrow \infty$ described in (ii) displays a strikingly unstable behavior. On the one hand, formally it is possible to construct a work extraction protocol that achieves Carnot efficiency for any number of particles $N$. On the other hand, this protocol has to be considered unphysical, since it requires that the external magnetic fields are controlled with degree of precision that scales with $N$. That is, for any finite precision on the external parameter $h(t)$, one can find a sufficiently large $N$ so that the maximal efficiency vanishes. This is discussed in detail in Sec. VI and in fact holds for more general classes of gapped ferromagnetic Hamiltonians. Since the precision on $h(t)$ is an intensive quantity, one can conclude that the strongly correlated ferromagnetic spin chains become useless as working mediums for engines in the thermodynamic limit. Indeed, we can also show that no finite work density can be achieved in the limit $J \rightarrow \infty$. Furthermore, this unstable behavior does not occur for the anti-ferromagnetic case (i). Lastly, to explain (iii) we derive that the form of the optimal protocol changes abruptly at $J=J^{*}$, explaining that the efficiency is not smooth in this point in the thermodynamic limit (see Sec. VIF).

\section{TECHNICAL RESULTS FOR THE GENERAL BOUND}

\section{A. Proof of Theorem 1}

The proof of Theorem 1 establishes a bound for the efficiency under any possible protocol that combines arbitrarily adiabatic operations Eq. (2) with thermal contacts with each of the baths Eq. (4), with the only constraint that the protocol is cyclic, in the sense that the initial and final Hamiltonian coincide. First, we show that the optimal protocol is of the form shown in Fig. 3(a). That is, the optimal protocol does not alternate contacts with the hot and the cold bath as in Fig. 3(b). Instead, one simply makes $n_{h}$ consecutive contacts with the hot bath, followed by $n_{c}$ contacts with the cold one. This family of protocols is referred to here as Carnot-like protocols and the first part of the proof is to show that they are optimal. 


\section{Optimality of Carnot-like protocols}

As mentioned above, a Carnot-like protocol is a cyclic process combining (i) $n_{h}$ consecutive thermal contacts with the hot bath and possibly adiabatic evolutions between the contacts and before the first and after the first contact with the hot bath, (ii) $n_{c}$ consecutive thermal contacts with the cold bath and possibly adiabatic evolutions between the contacts. Note that (without loss of generality) we always assume that the first sequence of thermal contacts is with the hot bath.

Let us consider now a noncyclic Carnot-like protocol. That is, a protocol that comprises steps (i) and (ii) but for which the initial and final Hamiltonian do not coincide. Let us denote by $H^{(i)}$ the initial Hamiltonian and $H^{(f)}$ the final one and also denote this noncyclic protocol by $\mathcal{P}^{\text {n.cy }}$. Clearly, we can add to the protocol $\mathcal{P}^{\text {n.cy }}$ an isothermal path from $H^{(f)}$ to $H^{(i)}$ (in contact with the cold bath), followed by another isothermal (also in contact with the cold bath) from $H^{(i)}$ to $H^{(f)}$ again. Similarly, we can complete this noncyclic protocol to a cyclic Carnot protocol $\mathcal{P}^{\text {cy }}$ by following $\mathcal{P}^{\text {n.cy }}$ by an isothermal from $H^{(f)}$ to $H^{(i)}$. Since isothermal paths are reversible, in the sense that

$$
W[\operatorname{isothermal}(i \rightarrow f)]+W[\operatorname{isothermal}(f \rightarrow i)]=0,
$$

we have that

$$
W\left(\mathcal{P}^{\text {cy }}\right)+W\left(\text { isothermal }(i \rightarrow f)=W\left(\mathcal{P}^{\text {n.cy }}\right) .\right.
$$

On the other hand, since we are adding isothermals in contact with the cold bath only, this does not affect the total heat extracted from the hot bath.

Let us now suppose we have an arbitrary cyclic protocol $\mathcal{P}$. Clearly, any protocol can be decomposed as a series, of, say $k$, noncyclic Carnot-like protocols $\mathcal{P}_{j}^{\text {n.cy }}$. Each of these noncyclic protocols has as initial Hamiltonian $H^{(j)}$ and as final $H^{(j+1)}$. Using Eq. (10) we obtain that the total work in $\mathcal{P}$ can be written as

$$
\begin{aligned}
W(\mathcal{P}) & =\sum_{j=1}^{k} W\left(\mathcal{P}_{j}^{\text {n.cy }}\right) \\
& =\sum_{j=1}^{k} W\left(\mathcal{P}_{j}^{\text {cy }}\right)+\sum_{j=1}^{k} W[\operatorname{isothermal}(j \rightarrow j+1)],
\end{aligned}
$$

where, since $\mathcal{P}$ is cyclic, we take $H^{(k+1)}=H^{(0)}$. The second term in the right-hand side of Eq. (12) is the work associated to a cyclic, reversible path. Using that isothermal paths are reversible we obtain that this term is equal to zero. Since the isothermal paths are only done while being in contact with the cold bath, be obtain also that $Q_{h}$ [isothermal $\left.(j \rightarrow j+1)\right]=0$ for all $j$, where $Q_{h}$ stands for the heat provided by the hot bath. Altogether, we obtain

$$
\begin{aligned}
& W(\mathcal{P})=\sum_{j=1}^{k} W\left(\mathcal{P}_{j}^{\mathrm{cy}}\right), \\
& Q_{h}(\mathcal{P})=\sum_{j=1}^{k} Q\left(\mathcal{P}_{j}^{\mathrm{cy}}\right) .
\end{aligned}
$$

The interpretation of the two previous equations is simply that any cyclic protocol has equal work and heat as a series of cyclic Carnot-like protocols, where no new Hamiltonians are introduced in the construction of the cyclic Carnot-like protocols. We will now show that the efficiency of a series of Carnot-like protocols is smaller than that of the most efficient of the Carnot-like protocols within the series. This will complete the proof that the optimal protocol is always a Carnot-like protocol. For simplicity let us consider here the case where $k=2$. In this case the protocol $\mathcal{P}$ can be decomposed as a series of two cyclic Carnot-like protocols with efficiency $\eta^{(1)}$ and $\eta^{(2)}$ respectively, so that $\eta^{(1)}>\eta^{(2)}$ (without loss of generality). Therefore, the total work and heat fulfill

$$
W(\mathcal{P}) \leqslant W^{(1)}+W^{(2)}, \quad Q(\mathcal{P})=Q_{h}^{(1)}+Q_{h}^{(2)} .
$$

This yields an efficiency bounded as

$$
\begin{aligned}
\eta(\mathcal{P}) & =\frac{W^{(1)}+W^{(2)}}{Q_{h}^{(1)}+Q_{h}^{(2)}} \\
& =\frac{\eta^{(1)} Q_{h}^{(1)}+\eta^{(2)} Q_{h}^{(2)}}{Q_{h}^{(1)}+Q_{h}^{(2)}} \\
& <\frac{\eta^{(1)} Q_{h}^{(1)}+\eta^{(1)} Q_{h}^{(2)}}{Q_{h}^{(1)}+Q_{h}^{(2)}} \\
& =\eta^{(1)},
\end{aligned}
$$

with $\eta^{(i)}=W^{(i)} / Q_{h}^{(i)}$. This calculation can be trivially extended to an arbitrary number of $k$ Carnot-like protocols in series. The efficiency is always lower than the optimal one of those Carnot-like protocols, which concludes the proof that Carnot-like protocols are optimal. The Hamiltonians appearing in Theorem 1 are then those of this optimal Carnot-like protocol.

\section{Optimal efficiency of a Carnot-like protocol}

Once we established the optimality of Carnot-like protocols, we will now calculate the optimal efficiency of a Carnotlike protocol. This will provide the optimal efficiency of an arbitrary protocol. We will make use for the proof of a result from Ref. [13]. There a similar set of operations is considered as the ones laid out in Sec. II. That is, unitary evolutions and thermal contacts, with the difference that only one thermal bath at inverse temperature $\beta$ is considered. It is shown that if the working medium is initially in state $\rho^{(i)}$ and Hamiltonian $H^{(i)}$ and finally in $\omega_{\beta}\left(H^{(f)}\right)$ and $H^{(f)}$, the work extracted in the process fulfills

$$
W^{i \rightarrow f} \leqslant \beta^{-1} D\left[\rho^{(i)} \| \omega_{\beta}\left(H^{(f)}\right)\right]-\beta^{-1} D\left[V \rho^{(i)} V^{\dagger} \| \omega_{\beta}\left(H^{*}\right)\right],
$$

where $V$ is any unitary operator that can be performed with the allowed set of Hamiltonians (given the control restriction) and $H^{*}$ is any allowed Hamiltonian. Equation (17) follows straightforwardly from Theorem 1 in Ref. [13].

Let us now compute the maximum efficiency of a Carnotlike protocol. We take the protocol depicted in Fig. 3(a) as an example of a Carnot-like protocol.

Let us assume (without loss of generality) that we start thermalized with the cold bath in Hamiltonian $H_{D}$. We then do an adiabatic evolution to the Hamiltonian $H_{A}$. After that we do the first thermal contact to the hot heat bath and then alternate 
between adiabatic evolutions and $n_{h}-1$ thermal contacts until we arrive at Hamiltonian $H_{B}$ in the state $\omega_{\beta_{h}}\left(H_{B}\right)$. In Fig. 3(a), the protocol depicted combines these $n_{h}$ contacts with the bath and the adiabatic evolutions to perform an isothermal path, but the following arguments apply in generality. From Eq. (17) we obtain that the total work from $H_{D}$ to $H_{B}$ is upper bounded as

$$
\begin{aligned}
W^{D \rightarrow B} \leqslant & F_{\beta_{h}}\left[\omega_{\beta_{c}}\left(H_{D}\right), H_{D}\right]-F_{\beta_{h}}\left[\omega_{\beta_{h}}\left(H_{B}\right), H_{B}\right] \\
& -T_{h} D\left[U \omega_{\beta_{c}}\left(H^{D}\right) U^{\dagger} \| \omega_{\beta_{h}}\left(H_{A}\right)\right] \\
=: & F_{\beta_{h}}\left[\omega_{\beta_{c}}\left(H_{D}\right), H_{D}\right]-F_{\beta_{h}}\left[\omega_{\beta_{h}}\left(H_{B}\right), H_{B}\right]-T_{h} C_{h}^{A} \\
= & \operatorname{tr}\left[H^{D} \omega_{\beta_{c}}\left(H^{D}\right)\right]-\operatorname{tr}\left[H^{B} \omega_{\beta_{h}}\left(H^{B}\right)\right] \\
& +T_{h}\left\{S\left[\omega_{\beta_{h}}\left(H^{B}\right)\right]-T_{h} S\left[\omega_{\beta_{c}}\left(H^{D}\right)\right]\right\}-T_{h} C_{h}^{A} \\
= & :-\Delta E^{D \rightarrow B}+T_{h}\left(S_{h}^{B}-S_{c}^{D}\right)-T_{h} C_{h}^{A} .
\end{aligned}
$$

Note that fixing the initial and final Hamiltonians $\left(H_{D}\right.$ and $\left.H_{B}\right)$ also fixes the initial and final states to be $\omega_{\beta_{c}}\left(H_{D}\right)$ and $\omega_{\beta_{h}}\left(H_{B}\right)$. In turn, this fixes the energy difference $\Delta E^{D \rightarrow B}$ between the initial and final states. Hence, from energyconservation, taking $Q_{h}^{D \rightarrow B}>0$ when energy leaves the hot bath, we get

$$
\Delta E^{D \rightarrow B}=-W^{D \rightarrow B}+Q_{h}^{D \rightarrow B} .
$$

With this we arrive at the upper bound,

$$
\begin{aligned}
Q_{h}^{D \rightarrow B} & \leqslant T_{h}\left\{S\left[\omega_{\beta_{h}}\left(H_{B}\right)\right]-S\left[\omega_{\beta_{c}}\left(H_{D}\right)\right]\right\}-T_{h} C_{h}^{A} \\
& =: T_{h}\left(S_{h}^{B}-S_{c}^{D}\right)-T_{h} C_{h}^{A} .
\end{aligned}
$$

After the contacts with the hot bath, we then perform an adiabatic evolution to $H_{C}$, followed by $n_{c}$ thermal contacts with the cold bath, with adiabatic evolutions in between, back to the initial Hamiltonian $H_{D}$. Completely analogous to the previous steps, the work done in this part is upper bounded as

$$
\begin{aligned}
W^{B \rightarrow D} \leqslant & -\Delta E^{B \rightarrow D}-T_{c}\left(S_{h}^{B}-S_{c}^{D}\right) \\
& -T_{c} D\left[V \omega_{\beta_{h}}\left(H_{B}\right) V^{\dagger} \| \omega_{\beta_{c}}\left(H_{C}\right)\right] \\
= & : \Delta E^{D \rightarrow B}-T_{c}\left(S_{h}^{B}-S_{c}^{D}\right)-T_{c} C_{c}^{C},
\end{aligned}
$$

and the heat from the cold bath is bounded as

$$
Q_{c}^{B \rightarrow D} \leqslant-T_{c}\left(S_{h}^{B}-S_{c}^{D}\right)-T_{c} C_{c}^{C} .
$$

The total work is then given by

$$
W(\mathcal{P}) \leqslant\left(T_{h}-T_{c}\right)\left(S_{h}^{B}-S_{c}^{D}\right)-T_{h} C_{h}^{A}-T_{c} C_{c}^{C} .
$$

Now note that the work can only be positive if the heat from the hot bath $Q_{h}(D \rightarrow B)$ is positive and that in this case the heat from the cold bath is negative. Using $Q_{h}+Q_{c}=W$ and $\eta(P):=Q_{h} / W$ we obtain

$$
\eta(\mathcal{P})=1-\frac{\left|Q_{c}\right|}{Q_{h}} \leqslant 1-\frac{T_{c}}{T_{h}}\left(\frac{\Delta S^{B, D}+C_{c}^{C}}{\Delta S^{B, D}-C_{h}^{A}}\right),
$$

with $\Delta S=S_{h}^{B}-S_{c}^{D}$. This proves the Theorem for Carnot-like protocols.

\section{B. Inequality regarding relative entropy}

We will now turn to proving the inequality

$$
D\left[U \omega_{\beta_{1}}\left(H_{1}\right) U^{\dagger} \| \omega_{\beta_{2}}\left(H_{2}\right)\right] \geqslant D^{\downarrow}\left[\omega_{\beta_{1}}\left(H_{1}\right) \| \omega_{\beta_{2}}\left(H_{2}\right)\right] .
$$

To do that we use the correspondence with the free energy and write the left-hand side as

$$
\begin{gathered}
\beta_{2} \operatorname{tr}\left[H_{2} U \omega_{\beta_{1}}\left(H_{1}\right) U^{\dagger}\right]-S\left(U \omega_{\beta_{1}} U^{\dagger}\right)+\log Z_{\beta_{2}}\left(H_{2}\right) \\
=\beta_{2} \operatorname{tr}\left[H_{2} U \omega_{\beta_{1}}\left(H_{1}\right) U^{\dagger}\right]-S\left(\omega_{\beta_{1}}\right)+\log Z_{\beta_{2}}\left(H_{2}\right) .
\end{gathered}
$$

The right-hand side corresponds to the case where $U$ is chosen such that $U \omega_{\beta_{1}}\left(H_{1}\right) U^{\dagger}$ is diagonal in the basis of $H_{2}$ with larger eigenvalues corresponding to smaller energies. It is thus the corresponds to the choice of $U$ that minimizes the energy. Hence, the left-hand side is always as least as big as the right-hand side.

\section{Carnot efficiency for vanishing interactions}

Observation 2 (Vanishing interactions). In the case of vanishing interactions, that is $H_{\text {int }}=0$, it is possible to achieve Carnot efficiency,

$$
\eta_{c}=1-\frac{T_{c}}{T_{h}} .
$$

This follows simply from Theorem 1 , since

$$
\omega_{h}^{B}:=\bigotimes_{i} \omega\left(H_{i}^{B}, \beta_{h}\right)=\bigotimes_{i} \omega\left(H_{i}^{C}, \beta_{c}\right):=\omega_{c}^{C}
$$

can be satisfied by taking simply $H_{i}^{C}=\left(\beta_{h} / \beta_{c}\right) H_{i}^{B}$, and equivalently for $\omega_{h}^{D}$ and $\omega_{h}^{A}$. By choosing $U=V=\mathbb{1}$ in the bound of Theorem 1, we obtain the Carnot efficiency Eq. (32).

\section{TECHNICAL RESULTS FOR THE ISING MODEL}

\section{A. Ground-state degeneracy}

In this section, we discuss the ground-state degeneracy of the nearest-neighbor Ising model with finite interactions and magnetic field. The goal is to explain the finite entropy density, and hence finite work density, that can be reached for any interaction strength in case of antiferromagnetic couplings. We will therefore restrict to this scenario. First, for any fixed temperature and zero magnetic field the thermal state converges to the thermal ground state as the interaction strength $J$ is made large in absolute value. Similarly, if the magnetic field has a strength $h=k J$, the thermal state approximates the ground state with unit interaction strength and magnetic field of strength $k$. For large interaction strengths, we are thus interested in the entropy density at zero temperature in the case $J=1$ and $h=k$. We therefore have to count the ground-state degeneracy in such a situation. Clearly, for $k \rightarrow \infty$ the groundstate degeneracy is finite, i.e., independent of the system size. The same holds for $k=0$ and an even number of spins while for $k=0$ and an odd number of spins, the ground-state degeneracy scales linearly with the system size. In all cases, the entropy density vanishes in the thermodynamic limit. We will now show that there are finite values of $k$ such that the ground-state degeneracy is exponentially large in the system size, so that the entropy density remains finite in the thermodynamic limit. 
To see this, suppose that we set $k$ to be given by the number of nearest neighbors of a single site in the lattice. For a square lattice we thus have $k=2 d$, with $d$ the spatial dimension. It is easy to convince oneself that one of the ground states is given by $|\uparrow\rangle^{\otimes N}$, where $|\uparrow\rangle$ denotes the spin-up state vector in the direction of the magnetic field. It has energy

$$
d N-2 d N=-d N .
$$

Now suppose that we flip one of the spins. The increase of energy due to the magnetic field is given by $2 k=4 d$, and the interaction energy of each of the $k$ neighbors is reduced by $2 J=2$. The net change of energy is found to be $2 k-2 k=0$, and we therefore have produced a new ground state. If we would flip a neighbor of the flipped spin, the energy would increase. However, if we flip a next-nearest neighbor of the flipped spin, we obtain a further ground state. Iterating in the same way we get new ground states until we have flipped half of the spins in the lattice. However, we can always decide to leave out one of the nearest neighbors. In other words, we can decide for each of the $N / 2$ next-nearest neighbors, whether we want to flip it, providing us with the lower bound of the ground-state entropy,

$$
S_{\mathrm{G}} \geqslant \log 2^{N / 2}=N / 2 \log 2 .
$$

Clearly, there are many more states with the same energy, for example, the one corresponding to $|\uparrow, \downarrow, \uparrow, \uparrow, \downarrow, \uparrow, \cdots\rangle$, which does not fit into the scheme described above. Nevertheless, our argument is sufficient to show that for $h=2 \mathrm{JZ}$, with $Z$ the coordination number of the lattice, we get a finite entropy density in the zero-temperature state in the thermodynamic limit (and hence also at any positive finite temperature).

\section{B. Achievability of Carnot efficiency in the antiferromagnet}

In this section, we show that Carnot efficiency is achievable at finite work per particle in the thermodynamic limit as $J \rightarrow$ $-\infty$, that is, in the extremely antiferromagnetic case. Recall that the work density in the thermodynamic limit is given by

$$
\begin{aligned}
w(J)= & \lim _{n \rightarrow \infty} \frac{1}{n}\left(T_{h}-T_{c}\right) \Delta S^{B, D} \\
& -\lim _{n \rightarrow \infty} \frac{1}{n}\left[T_{h} D\left(\omega_{\beta_{c}}^{H_{D}} \| \omega_{\beta_{h}}^{H_{A}}\right)+T_{c} D\left(\omega_{\beta_{h}}^{H_{B}} \| \omega_{\beta_{c}}^{H_{C}}\right)\right],
\end{aligned}
$$

with

$$
\Delta S^{B, D}=S\left(\omega_{\beta_{h}}^{H_{B}}\right)-S\left(\omega_{\beta_{c}}^{H_{D}}\right)
$$

Here, the Hamiltonians $H_{A, B, C, D}$ correspond to different magnetic fields $h_{A, B, C, D}$ at the different stages of the protocol. The efficiency is given by

$$
\eta(J)=1-\frac{T_{c}}{T_{h}} \frac{\Delta S^{B, D}+D\left(\omega_{\beta_{h}}^{H_{B}}|| \omega_{\beta_{c}}^{H_{C}}\right)}{\Delta S^{B, D}-D\left(\omega_{\beta_{c}}^{H_{D}}|| \omega_{\beta_{h}}^{H_{A}}\right)} .
$$

In the formula for the efficiency we have, for notational reasons, omitted the thermodynamic limit $n \rightarrow \infty$. Carnot efficiency is reached only if the two correction-terms involving relative entropies vanish [29]. The work density depends on $J$ and the external fields through the Hamiltonians $H_{A, B, C, D}$. In the limit $J \rightarrow-\infty$, the thermal states $\omega_{\beta}^{H}$ converge to ground-state projectors. We will now choose $h_{C}=h_{B}=2 J$ and $h_{D}=h_{A} \gg J \rightarrow \infty$ (compare this with the results of Sec. VIA). It is then clear that the relative entropy density including Hamiltonians $H_{A}$ and $H_{D}$ vanishes as it compares the state with all spins up with itself.

Similarly, in the limit $J \rightarrow-\infty$, the relative entropy involving $H_{B}=H_{C}$ vanishes, because the two states at different temperatures both converge to the ground state of a Hamiltonian with a finite $J<0$. Note, however, that in this case the two states converge to the ground state of a model in which $h=2 J$ and hence have finite entropy density.

Recall that the antiferromagnetic ground state at infinite external field has a unique ground state, whereas for $h=$ $2 J$, the ground-state space is exponentially degenerate in the system size and therefore has a finite entropy density (see Sec. VIA). Combining with the previous considerations, we have

$$
\begin{aligned}
\lim _{J \rightarrow-\infty} w(J) & =\lim _{J \rightarrow-\infty} \lim _{n \rightarrow \infty} \frac{1}{n}\left(T_{h}-T_{c}\right)\left(\Delta S^{B, D}\right) \\
& =\lim _{J \rightarrow-\infty} \lim _{n \rightarrow \infty} \frac{1}{n}\left(T_{h}-T_{c}\right) S\left(\omega_{\beta_{h}}^{H_{B}}\right) \\
& \geqslant\left(T_{h}-T_{c}\right) \frac{1}{2} \log (2),
\end{aligned}
$$

which is consistent with our numerics and the fact that we are in fact neglecting a number of ground states that is exponential in the system size in our estimate of the ground-state degeneracy.

Combining the fact that the entropy density $\Delta S^{B, D} / n$ remains finite with the observation that the corrections vanish, we obtain

$$
\lim _{J} \eta(J)=1-\lim _{J} \frac{T_{c}}{T_{h}} \frac{\Delta S^{B, D}}{\Delta S^{B, D}}=1-\frac{T_{c}}{T_{h}} .
$$

\section{Ferromagnetic case: Vanishing work for large $\boldsymbol{J}$}

In this section we show that in the limit $J \rightarrow \infty$ the optimal work density is zero for any value of $n$ and also in the thermodynamic limit. Let us first consider the case of arbitrary but finite $n$. From the discussion of Sec. VIA, we have that in the limit of large $J$ the thermal state approximates a projector on the lowest energy subspace. The dimension of the lowest energy subspace is equal to two, in the case of external fields $h=0$ (all spins facing up or down). If $h \neq 0$ the dimension is in turn one (all spins facing up or down depending on the sign of $h$ ). This implies that when $J \rightarrow \infty$ the optimal value of the entropy difference is $\Delta S^{B, D}=\log (2)$. Using Eq. (36), we obtain

$$
\begin{aligned}
\frac{W(J)}{n}= & \frac{1}{n}\left(T_{h}-T_{c}\right) \Delta S^{B, D} \\
& -\frac{1}{n}\left[T_{h} D\left(\omega_{\beta_{c}}^{H_{D}} \| \omega_{\beta_{h}}^{H_{A}}\right)+T_{c} D\left(\omega_{\beta_{h}}^{H_{B}} \| \omega_{\beta_{c}}^{H_{C}}\right)\right] \\
\leqslant & \frac{1}{n}\left(T_{h}-T_{c}\right) \log (2),
\end{aligned}
$$

where Eq. (42) follows from the optimal value of $\Delta S^{B, D}$ and the fact that the $D(\rho \| \sigma) \geqslant 0$ for all $\rho$ and $\sigma$.

One can easily see that this work output is indeed achievable for a finite $n$ in the limit of $J \rightarrow \infty$ for the choices $h_{A}=h_{D} \rightarrow \infty$ and $h_{B}=h_{C}=0$ in the sense that 
$h_{A}, h_{D} \gg J$ for any choice of $J$. We will show in Sec. VID that this protocol - which outputs optimal work for any value of $n$-has also null efficiency.

Last, we will also show that in the thermodynamic limit the work output vanishes in the limit of large $J$. That is, we will show that

$$
\lim _{J \rightarrow \infty} \lim _{n \rightarrow \infty} \frac{W(J)}{n} \leqslant \lim _{J \rightarrow \infty}\left(T_{h}-T_{c}\right) \Delta s^{B, C}=0,
$$

where $\Delta s^{B, C}=s_{\beta_{h}}\left(h_{B}\right)-s_{\beta_{c}}\left(h_{C}\right)$ and $s$ is the entropy per particle in the thermodynamic limit. Equation (43) is proven in Sec. VIC, where $s$ is explicitly computed for the Ising model.

\section{Ferromagnetic case: Vanishing efficiency}

We will now argue that the protocol providing optimal work for $J \rightarrow \infty$ put forward above not only has zero work yield, but also vanishing efficiency as $n$ is sufficiently large. As we will show, the efficiency of the protocol is related with the precision with which one can implement the magnetic fields $h_{B}=h_{C}=0$. In particular, we will show the following:

(1) If the protocol $h_{A}=h_{D} \rightarrow \infty$ and $h_{B}=h_{C}=0$ is implemented with exact precision, then the efficiency in the limit of $J \rightarrow \infty$ equals the Carnot efficiency.

(2) For any fixed finite precision $\epsilon$, so that $h_{B}=h_{C}=\epsilon$, there exist a sufficiently large $n$ so that for any $\delta$ the efficiency in the limit $J \rightarrow \infty$ is smaller than $\delta$.

That is, according to 1 it is formally possible to obtain Carnot efficiency in limit of strong coupling in the ferromagnetic case. However, 2 implies that to do so, we need to have a precision on the external magnetic fields which increases with $n$. From a practical point of view, the precision on the magnetic fields is determined by the functioning of the devices producing those fields and it is independent of the size of the working body on which the fields are applied. Hence, in a realistic experimental situation, one can conclude that the efficiency vanishes for sufficiently large $n$. In any case, regardless of the dependence of the precision and the size of the system, 2 implies that for any finite precision the efficiency vanishes in the thermodynamic limit.

We compute now the efficiency as a function of $J$ for the protocol $h_{A}=h_{D} \rightarrow \infty$ and $h_{B}=h_{C}=\epsilon$ with $\epsilon>0$, representing the precision with which we can control the magnetic field in the experiment. In the following it is also useful to keep in mind that the temperature-difference $\Delta T$ is fixed, and hence $\Delta T / J \rightarrow 0$, so that $\Delta T$ can be seen as arbitrarily small as $J$ goes to infinity. Now first notice that since $h_{A}, h_{D} \rightarrow \infty$, one of the penalty terms in the efficiency can effectively be set to zero just as in the antiferromagnetic case. Furthermore, $S\left(\omega_{\beta_{c}}^{H_{D}}\right)$ vanishes, so that $\Delta S^{B, D}$ can be replaced by $S\left(\omega_{\beta_{h}}^{H_{B}}\right)=: S_{h}^{B}$. The efficiency then takes the form

$$
\lim _{J} \eta(J)=\lim _{J}\left(1-\frac{T_{c}}{T_{h}} \frac{S_{h}^{B}+D\left(\omega_{\beta_{h}}^{H_{B}}|| \omega_{\beta_{c}}^{H_{B}}\right)}{S_{h}^{B}}\right) .
$$

We thus have to show that the second term converges to unity. To do that, first write the relative entropy as a difference of free energies and cancel entropic terms,

$$
\begin{aligned}
\frac{T_{c}}{T_{h}} \frac{S_{h}^{B}+D\left(\omega_{\beta_{h}}^{H_{B}} \| \omega_{\beta_{c}}^{H_{B}}\right)}{S_{h}^{B}} & =\frac{T_{c}}{T_{h}} \frac{S_{h}^{B}+\frac{1}{T_{c}}\left(E^{B}\left(T_{h}\right)-T_{c} S_{h}-F_{c}^{B}\right)}{S_{h}^{B}} \\
& =\frac{1}{T_{h}} \frac{E^{B}\left(T_{h}\right)-F_{c}^{B}}{S_{h}^{B}} \\
& =1+\frac{1}{T_{h}} \frac{F_{h}^{B}-F_{c}^{B}}{S_{h}^{B}} .
\end{aligned}
$$

Here, we have introduced the internal energy with respect to the Hamiltonian $H_{B}$ and at temperature $T$ as $E^{B}(T)$ and the thermal free energies at the temperatures $T_{h}$ and $T_{c}$ as $F_{h, c}^{B}$, respectively. They fulfill

$$
F_{h, c}^{B}=J f\left(T_{c, h} / J\right), \quad f(T):=-T \log Z_{T}(1, \epsilon / J),
$$

where $Z_{T}(J, h)$ is the partition function of the model at temperature $T$, interaction strength $J$, and magnetic field strength $h$. We can then expand $F_{h}^{B}$ in the small parameter $\left(T_{h}-T_{c}\right) / J=: \Delta T / J$ around $T_{c} / J$, to obtain

$$
\begin{aligned}
F_{h}^{B}-F_{c}^{B} & =J\left[\left.\frac{\partial f(x)}{\partial x}\right|_{x=T_{c} / J} \frac{T_{h}-T_{c}}{J}+O\left(1 / J^{2}\right)\right] \\
& =-S_{c}^{B}\left(T_{h}-T_{c}\right)+O(1 / J) .
\end{aligned}
$$

Using this result then yields for the efficiency

$$
\begin{aligned}
\lim _{j} \eta(J) & =\lim _{J \rightarrow \infty}\left[\frac{S_{c}^{B}}{S_{h}^{B}} \frac{\Delta T}{T_{h}}+O(1 / J)\right] \\
& \leqslant \frac{\Delta T}{T_{h}}\left[2 \frac{\beta_{c}}{\beta_{h}} e^{-\left(\beta_{c}-\beta_{h}\right) 2 c \epsilon N}+\frac{\log \left(1+e^{-\beta_{c} 2 \epsilon \epsilon N}\right)}{\log \left(1+e^{-\beta_{h} 2 c \epsilon N}\right)}\right],
\end{aligned}
$$

where $c>0$ is some constant and the last line is shown in the following section. We thus see that in any finite system it is formally possible to achieve Carnot-efficiency in the limit $J \rightarrow \infty$ if we can get $\epsilon$ exactly to zero as expressed by 1 . However, as the system size increases, to achieve a given efficiency, the error $\epsilon$ has to go to zero quicker than $1 / N$. If we have a fixed precision, the efficiency goes to zero exponentially, which proves 2 . We thus conclude that it is physically infeasible to achieve finite efficiency in the thermodynamic limit. In a finite system, in contrast, the efficiency in the limit of $J \rightarrow \infty$ depends on the precision $\epsilon$. This is illustrated in Fig. 4 for a system of six spins.

\section{E. Ratios of entropies}

In this section, we calculate the ratio of entropies

$$
\lim _{J \rightarrow \infty} \frac{S_{c}^{B}}{S_{h}^{B}} .
$$

Let us recall that $S_{c}^{B}$ is the entropy of the thermal states at inverse temperature $\beta_{c}$ for an external magnetic field $h=\epsilon$, and similarly for $S_{h}^{B}$ for a thermal state at inverse temperature $\beta_{h}$ and also external field $h=\epsilon$. The calculation holds for families of Hamiltonians more general than the Ising model. Hence, we compute it here in the general case for sake of 


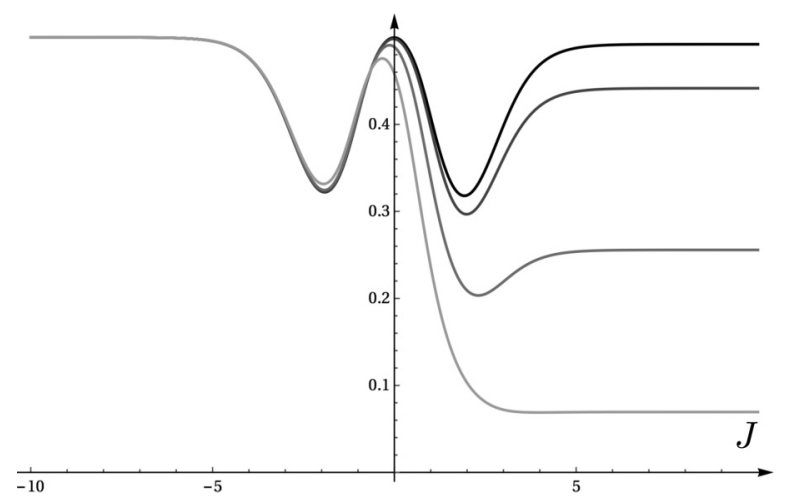

FIG. 4. Efficiency at maximum work for a system of six spins as a function of $J$ and different imprecisions on the external fields $\epsilon=0.05,0.1,0.25,0.5$ (black to light gray). For larger system sizes, the local minimum on the ferromagnetic side $J>0$ moves to larger values of $J$ and the value at $J=\infty$ decreases exponentially with the system size for any fixed precision.

generality. Let us consider a Hamiltonian of the form

$$
H=J H_{\text {int }}+\epsilon H_{\text {loc. }}=J\left(H_{\text {int }}+\frac{\epsilon}{J} H_{\text {loc. }}\right):=J H(J),
$$

with the following properties: We assume that (i) $H_{\text {int }}$ and $H_{\text {loc. }}$ are local Hamiltonians, (ii) the ground-state space of $H_{\text {int }}$ is twofold degenerate, (iii) $H_{\text {int }}$ has a spectral gap $\Delta$, that is, the energy difference with the smallest eigenvalue of $H_{\text {int }}$ other than the ground-state energy is $\Delta$. This condition on the degeneracy of the ground space is fulfilled by the Ising model as described in Sec. VI A. Let us know look at the lowest energy states of $H(J)$. In the limit of large $J$, we can treat $\frac{\epsilon}{J} H_{\text {loc. }}$ as a perturbation. Hence, the two ground states of $H_{\text {int }}$ are split by an gap $g$ that can be upper-bounded by the norm of the perturbation as

$$
g \leqslant\left\|\epsilon J^{-1} H_{\text {loc. }}\right\| \leqslant c n \frac{\epsilon}{J},
$$

where $c$ is some constant and $n$ the number of particles. In the following we assume that such splitting actually occurs and set $g=c n \frac{\epsilon}{J}$ for some $c>0$. Note that this assumption is fulfilled in the Ising-model if the perturbation is given by a homogeneous magnetic field. Altogether, taking the ground-state energy $E_{0}(J)$ to zero, we have that that the first excited level of $J H(J)$ is $E_{1}(J)=J g=c n \epsilon$ and the second excited level is $E_{2}(J) \geqslant J \Delta-g=J \Delta-O(1)$, where $O(1)$ indicates that is is constant on $J$. This given, we will now show that the entropy,

$$
S_{c / h}(J):=S\left\{\omega_{\beta_{c / h}}[J H(J)]\right\}=S\left[\omega_{\beta_{c / h}}\left(J H_{\text {int }}+\epsilon H_{\text {loc. }}\right)\right],
$$

fulfills the condition

$$
\lim _{J \rightarrow \infty} \frac{S_{c}(J)}{S_{h}(J)} \begin{cases}\leqslant 2 \frac{\beta_{c}}{\beta_{h}} e^{-\left(\beta_{c}-\beta_{h}\right) 2 c \epsilon n}+\frac{\log \left(1+e^{-\beta_{c} 2 \epsilon \epsilon n}\right)}{\log \left(1+e^{\left.-\beta_{h} 2 c \epsilon n\right)},\right.} & \epsilon \neq 0, \\ =1, & \epsilon=0 .\end{cases}
$$

To show the above let us also write $Z_{c / h}(J)=$ $\operatorname{tr}\left[e^{-\beta_{c / h} J H(J)}\right]$ for the partition function. Using the above conventions, it fulfills $Z_{c / h}(J) \geqslant 1$. We will use the formula

$$
S_{c / h}(J)=\beta_{c / h} E_{\beta_{c / h}}(J)+\log \left[Z_{c / h}(J)\right],
$$

with $E_{\beta_{c / h}}(J)$ the thermal energies of $J H(J)$ at inverse temperature $\beta_{c / h}$. Since $E_{2}(J)$ increases linearly with $J$, only the levels $E_{0}(J)$ and $E_{1}(J)$ contribute in the limit $J \rightarrow \infty$. We hence obtain

$$
\lim _{J \rightarrow \infty} S_{c / h}(J)=\beta_{c / h} \frac{2 c \epsilon n}{1+e^{-\beta_{c / h} 2 c \epsilon n}}+\log \left(1+e^{-\beta_{c / h} 2 c \epsilon n}\right) .
$$

We can then bound the ratio of entropies as

$$
\begin{aligned}
\lim _{J \rightarrow \infty} \frac{S_{c}(J)}{S_{h}(J)} & =\frac{\beta_{c} \frac{2 c \epsilon n}{1+e^{-\beta_{c} 2 c \epsilon n}}+\log \left(1+e^{-\beta_{c} 2 c \epsilon n}\right)}{\beta_{h} \frac{2 c \epsilon n}{1+e^{-\beta_{h} 2 c \epsilon n}}+\log \left(1+e^{-\beta_{h} 2 c \epsilon n}\right)} \\
& \leqslant 2 \frac{\beta_{c}}{\beta_{h}} e^{-\left(\beta_{c}-\beta_{h}\right) 2 c \epsilon n}+\frac{\log \left(1+e^{-\beta_{c} 2 c \epsilon n}\right)}{\log \left(1+e^{-\beta_{h} 2 c \epsilon n}\right)}
\end{aligned}
$$

where we have used in the last line that all the terms appearing are positive. On the other hand, by setting $\epsilon=0$ exactly, we obtain

$$
\lim _{J \rightarrow \infty} \frac{S_{c}(J)}{S_{h}(J)}=1,
$$

since both entropies converge to $\log (2)$.

\section{F. Optimal protocols in the thermodynamic limit \\ 1. Nonsmooth entropy at $J=J^{*}$}

In this section, we continue discussing the example of the Ising model and show that both the optimal work density and the efficiency at optimal work density are not smooth at $J=$ $J^{*}$. We will work directly in the thermodynamic limit, where the free-energy density takes the well-known form

$$
\begin{aligned}
f(\beta, J, h)= & -\frac{1}{\beta} \log \left\{e^{\beta J} \cosh (\beta h)\right. \\
& \left.+\left[e^{2 \beta J} \sinh (\beta h)^{2}+e^{-2 \beta J}\right]^{1 / 2}\right\} .
\end{aligned}
$$

It is clear from Eq. (36) that to optimize the work density, we have to maximize the entropy density as a function of the magnetic field for a given $J$. Here, we are interested in the antiferromagnetic regime, i.e., $J<0$. The entropy density can be calculated from the above expression explicitly by the usual formula,

$$
s(\beta, J, h)=-\frac{\partial}{\partial T} f(1 / T, J, h),
$$

resulting, however, in a fairly complicated expression. To find an extremum of the entropy density as a function of $h$, we take the corresponding derivative. The result is

$$
\begin{aligned}
& \frac{\partial s(\beta, J, h)}{\partial h} \\
& =-\beta^{2} \frac{e^{\beta J}[h \cosh (\beta h)+2 J \sinh (\beta h)]}{\left[e^{-2 \beta J}+e^{2 \beta J} \sinh (\beta h)^{2}\right]^{1 / 2}\left[1+e^{4 \beta J} \sinh (\beta h)^{2}\right]} .
\end{aligned}
$$

For this expression to vanish, we either need $h \rightarrow \infty$, so that the denumerator diverges, or that the numerator vanishes. The former case corresponds to vanishing entropy density, as it corresponds to a magnetic field that is so strong that it projects 


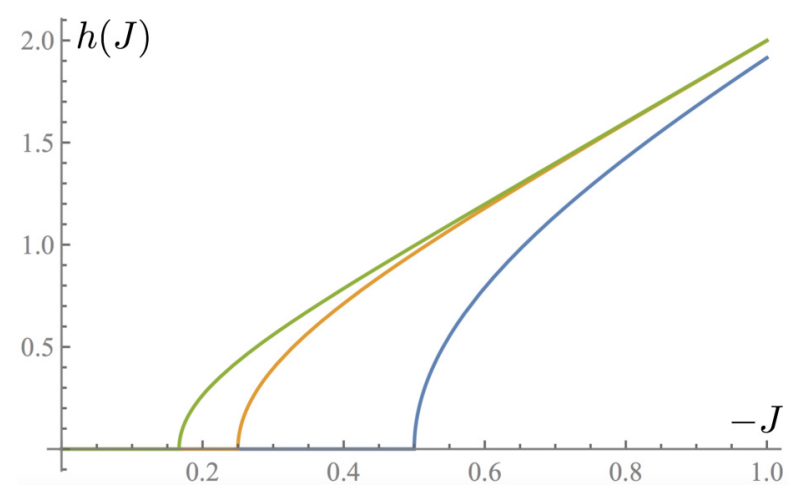

FIG. 5. The optimal magnetic field as a function of $J$ for inverse temperatures $\beta=1$ (blue), $\beta=2$ (orange), and $\beta=3$ (green). It is clearly visible that at the critical point $1 /(2 \beta)$ the function is not analytic, similarly to a second order phase transition.

all spins in the same direction. We thus consider the second case in which we have to find functions with the property that

$$
h(J) \cosh [\beta h(J)]+2 J \sinh [\beta h(J)]=0 .
$$

Clearly, one solution to this equation is given by $h_{1}(J)=$ 0 . However, there can be more solutions. Remembering that $J<0$, we can simplify this expression to

$$
h(J)=2|J| \tanh [\beta h(J)] .
$$

The existence of a second solution $h_{2}$ now follows from the fact that $h \mapsto \tanh (\beta h)$ is concave for $h>0$ and convex for $h<0$, with derivative at the origin given by $\beta>0$. Thus, as long as $2|J| \beta>1$, or, in other words,

$$
|J|>\frac{1}{2} k_{B} T
$$

there exists a second solution to the equation. It is also clear that this second solution only exists for $J<0$. We have plotted the optimal magnetic field in Fig. 5. It is clearly not continuously differentiable.

Finally, we note that the solution $h_{2}$ always provides a larger entropy than the trivial solution $h_{1}(J)=0$. From the discussion of the ground-state entropy in the antiferromagnetic case as a function of $h$, we can guess that for large $\beta$, the optimal magnetic field is given by $2|J|$. Indeed, we have

$$
2|J|[1-\tanh (\beta 2|J|)] \rightarrow 0
$$

as $|J| \rightarrow \infty$, showing that for very strong antiferromagnetic interactions $h(J)=2|J|$ is arbitrary close to the optimal value $h_{2}$.

\section{Optimal entropy difference in the ferromagnetic case}

We compute here the optimal entropy difference in the thermodynamic limit $\Delta s^{B, D}$ for $J \rightarrow \infty$. Indeed, we find that

$$
\lim _{J \rightarrow \infty} s(\beta, J, h)=0
$$

for all local fields $h$ and finite $\beta$. Hence, the difference $\Delta s^{B, D}=0$.

We can rewrite the free energy per particle $f(\beta, J, h)$ in Eq. (56)

$f(\beta, J, h)=-J-\frac{1}{\beta} \log \left[\cosh (\beta h)+\sqrt{e^{-4 \beta J}+\sinh (\beta h)^{2}}\right]$.

Using Eq. (57), we obtain

$$
\begin{aligned}
s(\beta, J, h)= & \log \left[\cosh (\beta h)+\sqrt{e^{-4 \beta J}+\sinh (\beta h)^{2}}\right] \\
& -\beta \frac{h \sinh (\beta h)+\frac{1}{2}\left[e^{-4 \beta J}+\sinh (\beta h)^{2}\right]^{-\frac{1}{2}}\left[-4 J e^{-4 \beta J}+2 h \sinh (\beta h) \cosh (\beta h)\right]}{\cosh (\beta h)+\sqrt{e^{-4 \beta J}+\sinh (\beta h)^{2}}} .
\end{aligned}
$$

Taking the limit of $J \rightarrow \infty$, we obtain

$$
\begin{aligned}
& \lim _{J \rightarrow \infty} s(\beta, J, h) \\
& =\log \left[\cosh (\beta h)+\sqrt{\sinh (\beta h)^{2}}\right] \\
& \quad-\beta \frac{h \sinh (\beta h)+\frac{1}{2}\left[\sinh (\beta h)^{2}\right]^{-\frac{1}{2}}[2 h \sinh (\beta h) \cosh (\beta h)]}{\cosh (\beta h)+\sqrt{\sinh (\beta h)^{2}}} \\
& =\log \left(e^{\beta h}\right)-\beta \frac{h[\cosh (\beta h)+\sinh (\beta h)]}{\cosh (\beta h)+\sinh (\beta h)}=0 .
\end{aligned}
$$

\section{CONCLUSION}

In this work, we have introduced the study of the performance of thermodynamic engines in the presence of local control on the thermodynamic operations. Limitations on control are ubiquitous in a wide range of thermodynamic processes. At the same time it is often the case that a very limited set of operations allows one to obtain maximal efficiency. Consider, for example, how restrictive it is to move a piston and put a gas in contact with two baths-which suffices to reach the optimal efficiency-when compared with all conceivable operations-which might include acting on the individual molecules of the gas. In other words, it is often the case that when it comes to work extraction, a severe restriction in the operations does not compromise the efficiency. In contrast, here, we find that the very reasonable limitation of having local control results in a reduction of the efficiency in full generality. In particular, we derived bounds to Carnot efficiency and showed that these bounds are achievable for generic interactions. This opens new venues to incorporate in a comprehensive fashion the extensive literature on quantum control to thermodynamics.

Possible extensions of our formalism include more general and realistic thermal baths, as well as local unitaries instead of 
local external fields. Last, the corrections on the efficiency can be investigated for systems other than the 1D-Ising model. In particular, it would be interesting to understand the effects on the efficiency for systems displaying spontaneous magnetization for low temperatures.

\section{ACKNOWLEDGMENTS}

We acknowledge funding from the BMBF (Q.com), the EU (RAQUEL, AQuS), the DFG (EI 519/7-1, CRC 183, GA 2184/2-1), the ERC (TAQ), and the Studienstiftung des Deutschen Volkes.
[1] N. Linden, S. Popescu, and P. Skrzypczyk, How Small Can Thermal Machines Be? The Smallest Possible Refrigerator, Phys. Rev. Lett. 105, 130401 (2010).

[2] L. del Rio, J. Aberg, R. Renner, O. Dahlsten, and V. Vedral, The thermodynamic meaning of negative entropy, Nature 474, 61 (2011).

[3] J. Aberg, Truly work-like work extraction via a single-shot analysis, Nat. Commun. 4, 1925 (2013).

[4] A. Mari and J. Eisert, Cooling by Heating: Very Hot Thermal Light Can Significantly Cool Quantum Systems, Phys. Rev. Lett. 108, 120602 (2012).

[5] F. G. S. L. Brandao, M. Horodecki, N. H. Y. Ng, J. Oppenheim, and S. Wehner, The second laws of quantum thermodynamics, Proc. Natl. Acad. Sci. USA 112, 3275 (2015).

[6] F. G. S. L. Brandão, M. Horodecki, J. Oppenheim, J. M. Renes, and R. W. Spekkens, The Resource Theory of Quantum States out of Thermal Equilibrium, Phys. Rev. Lett. 111, 250404 (2013).

[7] R. Alicki, The quantum open system as a model of the heat engine, J. Phys. A 12, L103 (1979).

[8] R. Kosloff, A quantum mechanical open system as a model of a heat engine, J. Chem. Phys. 80, 1625 (1984).

[9] J. Gemmer, M. Michel, and G. Mahler, Quantum Thermodynamics (Springer, Berlin, 2009).

[10] D. Gelbwaser-Klimovsky, W. Niedenzu, and G. Kurizki, Thermodynamics of quantum systems under dynamical control, Adv. At. Mol. Opt. Phys. 64, 329 (2015).

[11] C. Perry, P. Ćwikliński, J. Anders, M. Horodecki, and J. Oppenheim, A sufficient set of experimentally implementable thermal operations, arXiv:1511.06553.

[12] M. Lostaglio, Á. M. Alhambra, and C. Perry, Elementary thermal operations, Quantum 2, 52 (2018).

[13] H. Wilming, R. Gallego, and J. Eisert, Second law of thermodynamics under control restrictions, Phys. Rev. E 93, 042126 (2016).

[14] J. Oppenheim, M. Horodecki, P. Horodecki and R. Horodecki, Thermodynamical Approach to Quantifying Quantum Correlations, Phys. Rev. Lett. 89, 180402 (2002).

[15] R. Alicki and M. Fannes, Entanglement boost for extractable work from ensembles of quantum batteries, Phys. Rev. E 87, 042123 (2013).
[16] K. V. Hovhannisyan, M. Perarnau-Llobet, M. Huber, and A. Acín, Entanglement Generation is Not Necessary for Optimal Work Extraction, Phys. Rev. Lett. 111, 240401 (2013).

[17] M. Perarnau-Llobet, K. V. Hovhannisyan, M. Huber, P. Skrzypczyk, N. Brunner, and A. Acin, Extractable Work from Correlations, Phys. Rev. X 5, 041011 (2015).

[18] M. Campisi and R. Fazio, The power of a critical heat engine, Nat. Commun. 7, 11895 (2016).

[19] M. Campisi and R. Fazio, Dissipation, correlation, and lags in heat engines, J. Phys. A: Math. Theor. 49, 345002 (2016).

[20] Y. Zheng and D. Poletti, Quantum statistics and the performance of engine cycles, Phys. Rev. E 92, 012110 (2015).

[21] D. Janzing, P. Wocjan, R. Zeier, R. Geiss, and T. Beth, Thermodynamic cost of reliability and low temperatures: Tightening Landauer's principle and the second law, Int. J. Theor. Phys. 39, 2717 (2000).

[22] If the subsystems do not interact-or they do it in the weakcoupling regime - the set of possible dynamics is trivially given by products of local unitaries. In this regime the limitations of local control are not present.

[23] S. Lloyd, Almost Any Quantum Logic Gate is Universal, Phys. Rev. Lett. 75, 346 (1995).

[24] V. Ramakrishna, M. V. Salapaka, M. Dahleh, H. Rabitz, and A. Peirce, Controllability of molecular systems, Phys. Rev. A 51, 960 (1995).

[25] F. Albertini and D. D'Alessandro, The Lie algebra structure and controllability of spin systems, Linear Algebra Appl. 350, 213 (2002).

[26] D. Janzing, F. Armknecht, R. Zeier, and T. Beth, Quantum control without access to the controlling interaction, Phys. Rev. A 65, 022104 (2002).

[27] S. Lloyd, A. J. Landahl, and J.-J. E. Slotine, Universal quantum interfaces, Phys. Rev. A 69, 012305 (2004).

[28] D. Burgarth, S. Bose, C. Bruder, and V. Giovannetti, Local controllability of quantum networks, Phys. Rev. A 79, 060305(R) (2009).

[29] Indeed, this condition is not sufficient, as we will see in the ferromagnetic case. 